\title{
Time scales of the slowly drifting pulsating structure observed during the April 12, 2001 flare
}

\author{
M. Karlický ${ }^{1}$, M. Bárta ${ }^{1}$, H. Mészárosová ${ }^{1}$, and P. Zlobec ${ }^{2}$ \\ 1 Astronomical Institute of the Academy of Sciences of the Czech Republic, 25165 Ondřejov, Czech Republic \\ e-mail: karlicky@asu.cas.cz \\ 2 INAF - Trieste Astronomical Observatory, via G.B. Tiepolo 11, 34131 Trieste, Italy
}

Received 29 June 2004 / Accepted 1 November 2004

\begin{abstract}
First time scales of high-frequency (500-1500 MHz) slowly drifting pulsating structures observed during the April 12, 2001 flare by the Ondřejov ( $800-4500 \mathrm{MHz})$ and Potsdam (40-800 MHz) radiospectrographs and by the 1420 and $610 \mathrm{MHz}$ Trieste radiopolarimeters (with high time resolution $(1 \mathrm{~ms})$ ) are studied statistically. The Fourier method reveals periods in the range of 0.9-7.5 s. For shorter periods the power spectra show a power-law form, especially in the interval of about $0.06-0.2 \mathrm{~s}$, where the power-law index is in the 1.3-1.6 range. The results are interpreted using the flare model with plasmoid ejections. For the first time, the multi-scale cascading reconnection process is included in the interpretation. Corresponding time scales are estimated analytically. Further, magnetic reconnection in the bursting regime is simulated in a 2-D MHD model and variations of the dissipation power and radio radiation measure are computed. Fourier spectra of these numerical variations are determined and compared with those obtained from observations.
\end{abstract}

Key words. plasmas - Sun: flares - Sun: radio radiation

\section{Introduction}

Slowly drifting pulsating structures are observed in the $0.6-1.5 \mathrm{GHz}$ frequency range in association with plasmoid ejection at the beginning of some eruptive solar flares (Karlický \& Odstrčil 1994; Kliem et al. 2000; Hudson et al. 2001; Kundu et al. 2001; Khan et al. 2002; Karlický et al. 2002).

The papers by Karlický \& Odstrčil (1994), Hudson et al. (2001), Kundu et al. (2001), Khan et al. (2002), Karlický et al. (2002) presented observational details of various drifting pulsating structures; Kliem et al. (2000) proposed a theoretical model. Based on MHD numerical simulations, Kliem et al. (2000) suggested that each individual burst in the slowly drifting pulsating structure is generated by a beam of superthermal electrons, accelerated in the peak of the electric field in the quasi-periodic and bursting regime of magnetic field reconnection. The global slow negative frequency drift of the structure was explained by a plasmoid propagation upwards in the solar corona towards lower plasma densities.

Tanuma et al. (2001) and Shibata \& Tanuma (2001) have shown that due to the tearing mode instability, the reconnection process has a multi-scale (cascading) form: many plasmoids of different spatial and time scales are formed. Moreover, the plasmoids can merge into bigger ones. Thus, it can be expected that these plasmoids can be "visualized" in radio waves by accelerated electron beams, i.e. several slowly drifting structures could be observed during one solar flare. Radio observations presented in the paper by Karlický (2004) confirm such an expectation. This has led to the suggestion that a series of slowly drifting structures map the reconnection process.

In the present paper, analyzing unique high resolution data and considering the abovementioned concept of multi-scale reconnection, we extend the analysis made by Kliem et al. (2000). Combining the 1420 and $610 \mathrm{MHz}$ high-time resolution (1 ms) and 40-4500 MHz radio spectral observations, we analyze the characteristic time scales of observed slowly drifting structures (Sect. 3). In Sect. 4 the results are interpreted in the flare model with a plasmoid ejection; analytical estimations of time scales of the proposed multi-scale reconnection are also given. Fourier spectra of plasma parameters in the 2-D MHD model of reconnection are computed (Sect. 5) and compared with those observed (Sect. 6).

\section{Observations}

On April 12, 2001 the GOES satellite observed a soft X-ray event which started at 09:39 UT, with the maximum at 10:28 and end at 10:49. This event was classified as X2.0 and localized in the active region NOAA AR 9415.

Figure 1 shows the radio spectrum of this flare in a broad range of frequencies from 40 to $4500 \mathrm{MHz}(40-800 \mathrm{MHz}$ observed by the Potsdam and $800-4500 \mathrm{MHz}$ by the Ondřejov radiospectrographs). In radio waves the flare started at 10:14:30 at about $1500 \mathrm{MHz}$ with a series of fast drifting bursts covering 


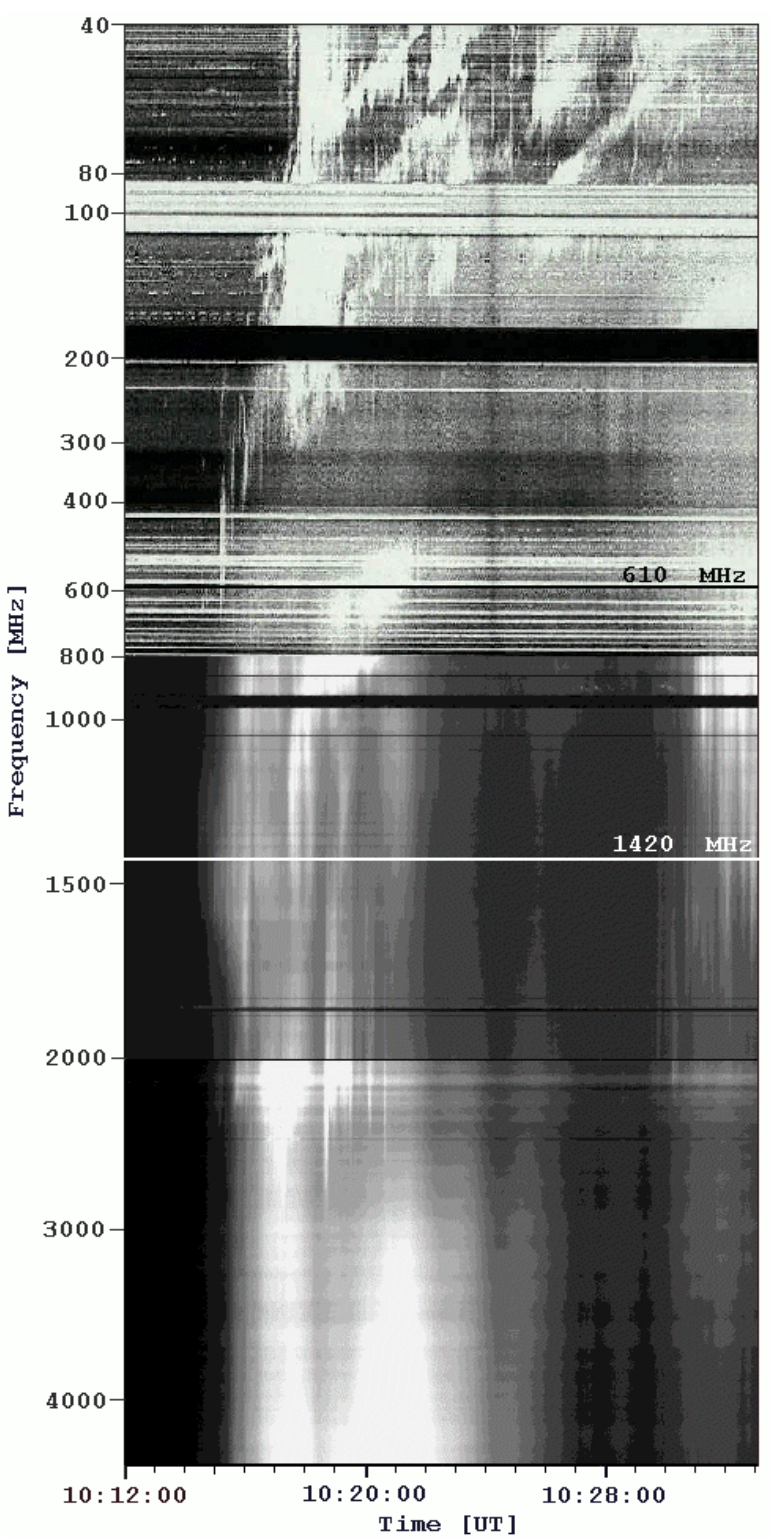

Fig. 1. 40-4500 $\mathrm{MHz}$ radio spectrum observed by Potsdam (40-800 MHz range, courtesy by $\mathrm{A}$. Klassen) and Ondřejov radiospectrographs, showing the slowly drifting pulsating structure at 10:17:20-10:22:00 UT in the 450-1500 $\mathrm{MHz}$ range and the type II radio burst at 10:17-10:33 in the $40-300 \mathrm{MHz}$ range. The Trieste observing frequencies $(610$ and $1420 \mathrm{MHz}$ ) are shown by horizontal lines (black and white, respectively).

the band from 200 to at least $4500 \mathrm{MHz}$. Below $800 \mathrm{MHz}$ these bursts formed a precursor which was followed by the type II burst at 10:17-10:33 in the metric range (Klassen et al. 2003).

From 10:16:00-10:17:30 and 10:17:20-10:22:00 in the range $450-1000 \mathrm{MHz}$ and $450-1500 \mathrm{MHz}$ respectively, highfrequency slowly drifting pulsating structures were observed. The second one was stronger and its global frequency drift was about $-1.8 \mathrm{MHz} \mathrm{s}^{-1}$. The time evolution of the X-ray $\mathrm{HXT} /$ Yohkoh $(33-53 \mathrm{keV})$ and $3 \mathrm{GHz}, 1420 \mathrm{MHz}$ and $610 \mathrm{MHz}$ radio fluxes at the very beginning of the flare is shown in Fig. 2, where the start of the precursor, the weak and the strong high-frequency drifting pulsating structures are

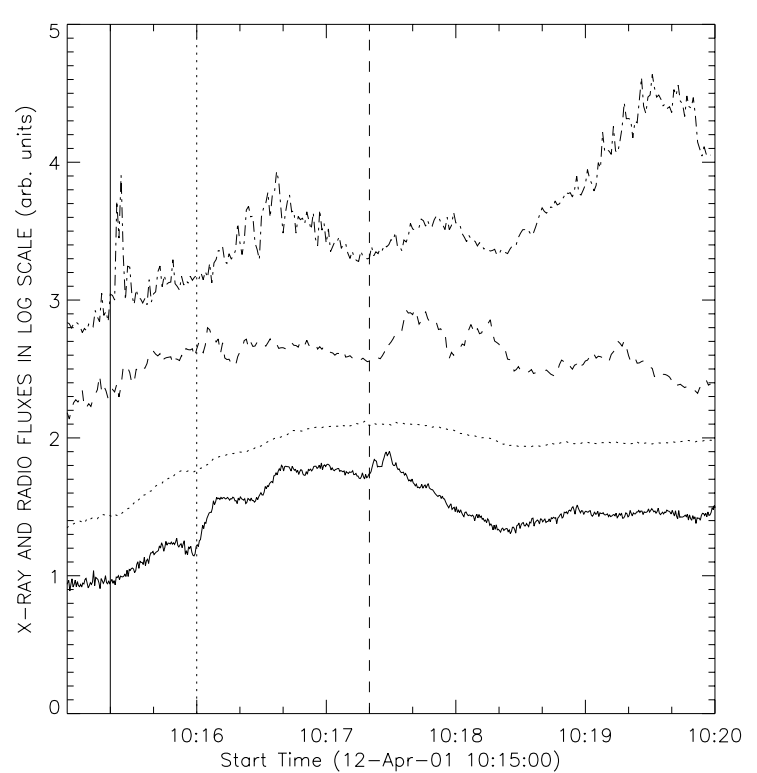

Fig. 2. X-ray and radio fluxes observed at the beginning of the April 12, 2001 event. The full line expresses the $33-53 \mathrm{keV}$ X-ray flux, the dotted line the $3 \mathrm{GHz}$ flux, the dashed line the $1420 \mathrm{MHz}$ and dashed-dotted line $610 \mathrm{MHz}$ right circular polarized radio fluxes. Vertical lines show starts of spectral features: the full line shows the start of the type II burst precursor, the dotted line the start of a weak drifting pulsating structure and the dashed line the start of the strong drifting pulsating structure.

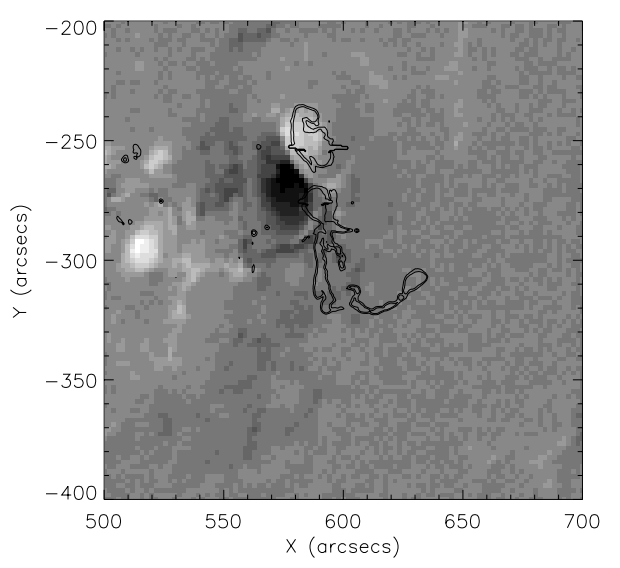

Fig. 3. TRACE $1600 \AA$ image observed at 10:16:11 (contours) superposed on the MDI/SOHO line-of-sight magnetic field observed at 09:36:02 (grey scale). White areas correspond to positive magnetic field polarity.

expressed by full, dotted and dashed vertical lines, respectively. As can be seen here, there is a close time coincidence between hard-X ray and radio flux enhancements.

The flare was observed also by the EIT/SOHO experiment in the 1600 and $171 \AA$ lines. The image at 10:16:11 (1600 $\AA$ ) superposed on the MDI/SOHO magnetogram, observed at 09:36:02, is presented in Fig. 3. Bright flare features had a sigmoid form elongated along the neutral line between opposite magnetic field polarities.

The radio emission of the flare was also recorded at two single frequencies (610 and $1420 \mathrm{MHz}$ ) by the Trieste radiopolarimeter with $1 \mathrm{~ms}$ time resolution (Fig. 4). While the 


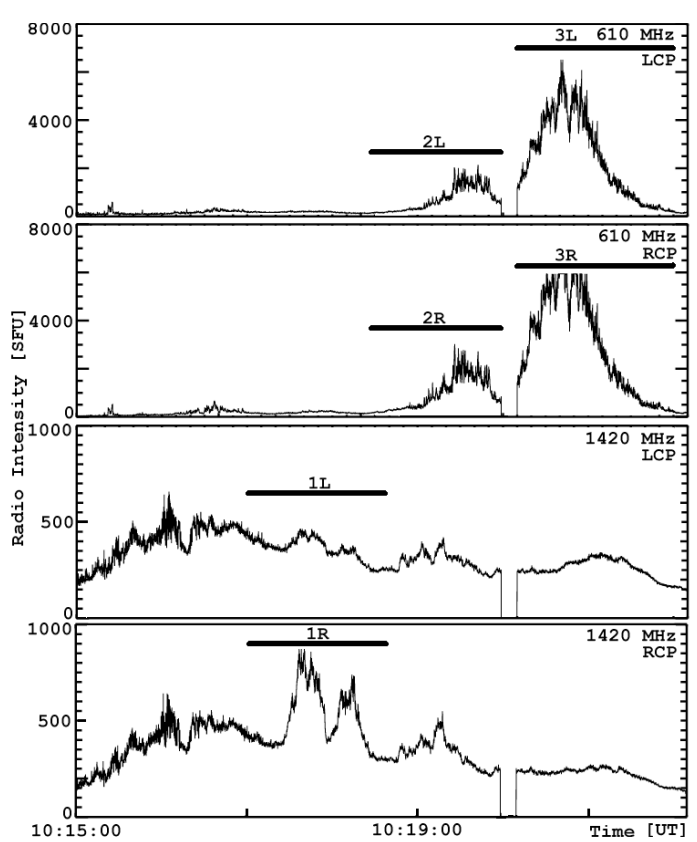

Fig. 4. Left (L) and right (R) circular polarization of the April 12, 2001 fluxes observed at 1420 and $610 \mathrm{MHz}$ by the Trieste radiopolarimeter. The intervals studied statistically are designated by numbers 1,2 and 3 .

beginning of the flare is practically unpolarized, at times when the single frequency observations intersect the second, i.e. stronger slowly drifting structure (10:17:20-10:18:30 at $1420 \mathrm{MHz}$ and 10:18:30-10:22:00 at $610 \mathrm{MHz})$ the radio emission is polarized in the right-circular sense. The mean polarization at these times is $50-60 \%$ at $1420 \mathrm{MHz}$ and $10-20 \%$ at $610 \mathrm{MHz}$, respectively.

\section{Analysis of time scales}

For the statistical analysis three intervals containing the stronger slowly drifting structure at 1420 and $610 \mathrm{MHz}$ were selected (Fig. 4, intervals designated 1R, 1L, 2R, 2L, 3R, 3L; $\mathrm{R}$ and $\mathrm{L}$ mean right and left circular polarization, respectively). (The receiver at $610 \mathrm{MHz}$ was saturated when the flux density reached about $6000 \mathrm{SFU}$, which is more evident in the R-channel.) We used the Fourier method. Power spectra for selected intervals are shown in Fig. 5, after the subtraction of the spectrum obtained during pre-flare interval. We recognized periods in the range of seconds $(0.9-7.5 \mathrm{~s})$ with high statistical probability (86-95\%); they are summarized in Table 1. In shorter periods, many individual periods in the range of $\sim 0.2-0.9 \mathrm{~s}$ can be seen; in the range of $0.2-0.06 \mathrm{~s}$, Fourier spectra show a power-law form $\left(\sim f^{-\gamma}\right)$ with a power-law index $\gamma$ in the 1.3-1.6 range (see Table 1). For even shorter periods $(<0.06 \mathrm{~s})$ only noise was present (Fig. 5).

The data in the intervals $1 \mathrm{R}, 1 \mathrm{~L}, 2 \mathrm{R}, 2 \mathrm{~L}$ and in the nonsaturated parts of the intervals $3 \mathrm{R}$ and $3 \mathrm{~L}$ were tested for lowdimensional determinism and nonlinearity (surrogate data tests and correlation dimensions, for the methods see Mészárosová et al. 2003). Non-linear behaviour was found in the nonsaturated parts of the $3 \mathrm{R}$ and $3 \mathrm{~L}$ intervals, which indicates that the generating process can be described by non-linear

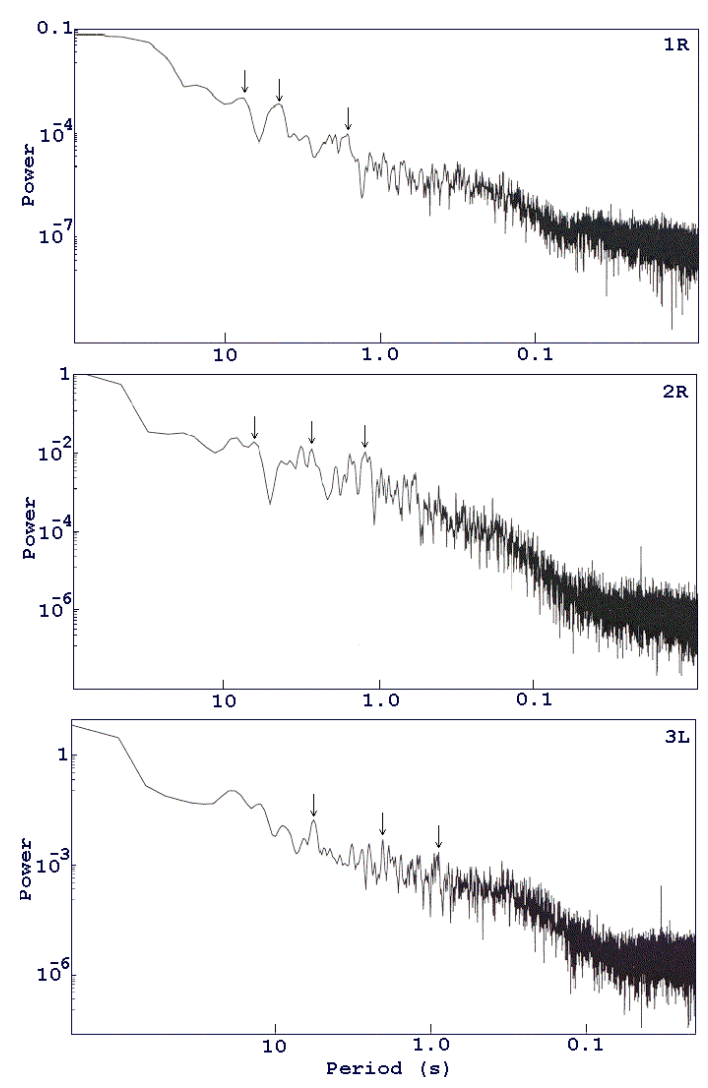

Fig. 5. Fourier power spectra in three selected intervals $1 \mathrm{R}, 2 \mathrm{R}, 3 \mathrm{~L}$ during the drifting structures (see Fig. 4). Fourier power spectra made in the interval before the bursts (radio flux of "quiet sun") were subtracted. The arrows show the periods reported in Table 1 .

equations. All intervals indicated high dimensional or stochastic processes.

\section{Interpretation of time scales}

Based on the paper by Kliem et al. (2000) we propose that during a bursting reconnection local enhancements of the electric field are generated and simultaneously electron beams are accelerated. These beams generate pulses inside slowly drifting pulsating structures through the bump-on-tail instability and wave transformation. The pulses are spatially localized (i.e. localized also in the radiation frequency) due to trapping of beams in the O-type magnetic field structures (plasmoids). The partial right-circular polarization of the high-frequency structure argues in favour of radio emission at the fundamental plasma frequency.

In such a scenario we propose that spatial scales of plasmoids in the current sheet correspond to those generated by a cascade of tearing instabilities - multi-scale reconnection (Shibata \& Tanuma 2001). On the other hand, time scales of enhanced electric fields, localized between neighbouring plasmoids, follow from their subsequent coalescence processes as described by Tajima et al. (1987).

Now, let us explain these processes in more detail: in agreement with Shibata \& Tanuma (2001) we assume that the magnetic flux rope moves upwards and below it the current sheet is formed. The length of the current sheet $L_{0}(t)$ grows in time $t$ 
Table 1. Frequencies, analyzed intervals, periods and power-law indexes. For periods, see the arrows in Fig. 5.

\begin{tabular}{cccc}
\hline \hline Interval (see Fig. 4) & $1 \mathrm{R}$ & $2 \mathrm{R}$ & $3 \mathrm{~L}$ \\
Frequency (MHz) & 1420 & 610 & 610 \\
Time interval & $10: 17: 10-10: 18: 40$ & $10: 18: 30-10: 19: 59$ & $10: 20: 10-10: 22: 00$ \\
Periods (s) & $7.5,4.5,1.6$ & $6.3,2.7,1.2$ & $5.7,2.0,0.9$ \\
Probabilities & $95,88,94$ & $95,94,95$ & $86,87,89$ \\
of periods (\%) & & & 1.3 \\
Power-law index $\gamma$ & 1.4 & 1.6 & \\
of Fourier spectrum & & & $0.3-0.06$ \\
in period range (s) & $0.2-0.06$ & $0.2-0.06$ & \\
\hline
\end{tabular}

and at the moment when the growth time of the tearing mode instability $t_{\text {tear }}$ becomes lower than the time for the reconnection flow to carry the perturbation out of the current sheet, i.e. at

$t_{\text {tear }} \leq L_{0}(t) / v_{\mathrm{A}}$,

where $v_{\mathrm{A}}$ is the Alfvén speed, the current sheet becomes unstable for the tearing instability. The growth time and wavelength of the tearing instability can be expressed as (Priest \& Forbes 2000)

$t_{\text {tear }} \simeq\left(\frac{l_{0}^{3}}{\eta v_{\mathrm{A}}}\right)^{1 / 2}$,

$L_{1} \simeq 2 \pi l_{0}\left(\frac{l_{0} v_{\mathrm{A}}}{\eta}\right)^{1 / 4}$,

where $l_{0}$ is the initial thickness of the current sheet, $\eta$ is the magnetic diffusivity. Now expressing $l_{0}$ by $L_{0}$ (using the relations (1) and (2)), the relation (3) gives

$L_{1} \simeq 6 \eta^{1 / 6} v_{\mathrm{A}}^{-1 / 6} L_{0}^{5 / 6}=A L_{0}$,

where $A=6 R_{\mathrm{m}}^{-1 / 6}, R_{\mathrm{m}}=L_{0} v_{\mathrm{A}} / \eta$ is the magnetic Reynolds number. During this first tearing instability in its non-linear phase at places between O-type structures the current sheet becomes thinner and further tearing instability starts. This process continues in shorter and shorter spatial scales - a cascade of tearing instabilities occurs. Thus the characteristic scale at the $n+1$ level of instability can be written as a generalized form of previous relation

$\frac{L_{\mathrm{n}+1}}{L_{0}} \simeq A\left(\frac{L_{\mathrm{n}}}{L_{0}}\right)^{5 / 6}$,

$\frac{L_{\mathrm{n}+1}}{L_{0}} \simeq A^{6(1-x)}$,

where $x=(5 / 6)^{n+1}$.

After the cascading process, which according to Shibata \& Tanuma (2001) lasts

$t_{\text {total }} \leq t_{0} A_{0}^{3 / 2}$,

where

$t_{0}=\frac{l_{0}^{3 / 2}}{\left(\eta v_{\mathrm{A}}\right)^{1 / 2}}$,

$A_{0}=6^{3 / 2}\left(\frac{\eta}{l_{0} v_{\mathrm{A}}}\right)^{1 / 6}$ plasmoids of different spatial scales (relation (5)) appeared in the current sheet.

On the other hand, these plasmoids can interact and through dissipative processes energy can be released. If we consider the interaction between two plasmoids of the same size then the characteristic time scale for this interaction is (see Tajima et al. 1987, and their relation (34))

$t^{n} \approx 2 \pi \beta^{3 / 2} \frac{L_{\mathrm{n}}}{v_{\mathrm{A}}}$,

where $\beta$ is the plasma $\beta$ parameter - the ratio of the kinetic to the magnetic energy densities. A broad range of spatial scales of plasmoids thus gives a broad range of time intervals in which the flare energy can be released.

\section{Numerical simulations}

Now, let us simulate the proposed reconnection processes in order to compare their spectral characteristics with those found by Fourier analysis of the observed data.

The 2-D MHD model similar to those in Karlický (1988), Schumacher \& Kliem (1996), and Kliem et al. (2000) is used. The model MHD equations are:

$\partial_{t} \rho=-\nabla \cdot(\rho \boldsymbol{u})$

$\rho \partial_{t} \boldsymbol{u}=-\rho(\boldsymbol{u} \cdot \nabla) \boldsymbol{u}-\nabla p+\boldsymbol{j} \times \boldsymbol{B}$,

$\partial_{t} \boldsymbol{B}=\nabla \times(\boldsymbol{u} \times B)-\nabla \times(\eta \boldsymbol{j})$,

$\partial_{t} U=-\nabla \cdot S$,

where the current density $\boldsymbol{j}$, the total energy density $U$, and the flux vector $\boldsymbol{S}$ are given by

$\boldsymbol{j}=\frac{1}{\mu_{0}} \nabla \times \boldsymbol{B}$,

$U=\rho w+\frac{\rho}{2} u^{2}+\frac{B^{2}}{2 \mu_{0}}$,

$\boldsymbol{S}=\left(U+p+\frac{B^{2}}{2 \mu_{0}}\right) \boldsymbol{u}-(\boldsymbol{u} \cdot \boldsymbol{B}) \frac{\boldsymbol{B}}{\mu_{0}}+\eta \boldsymbol{j} \times \frac{\boldsymbol{B}}{\mu_{0}}$,

and $w$ is the internal energy per unit mass, which is related to the pressure through the equation of state, $p=(\gamma-1) \rho w$. The ratio of specific heats is $\gamma=(N+2) / N=2$, where $N$ is 

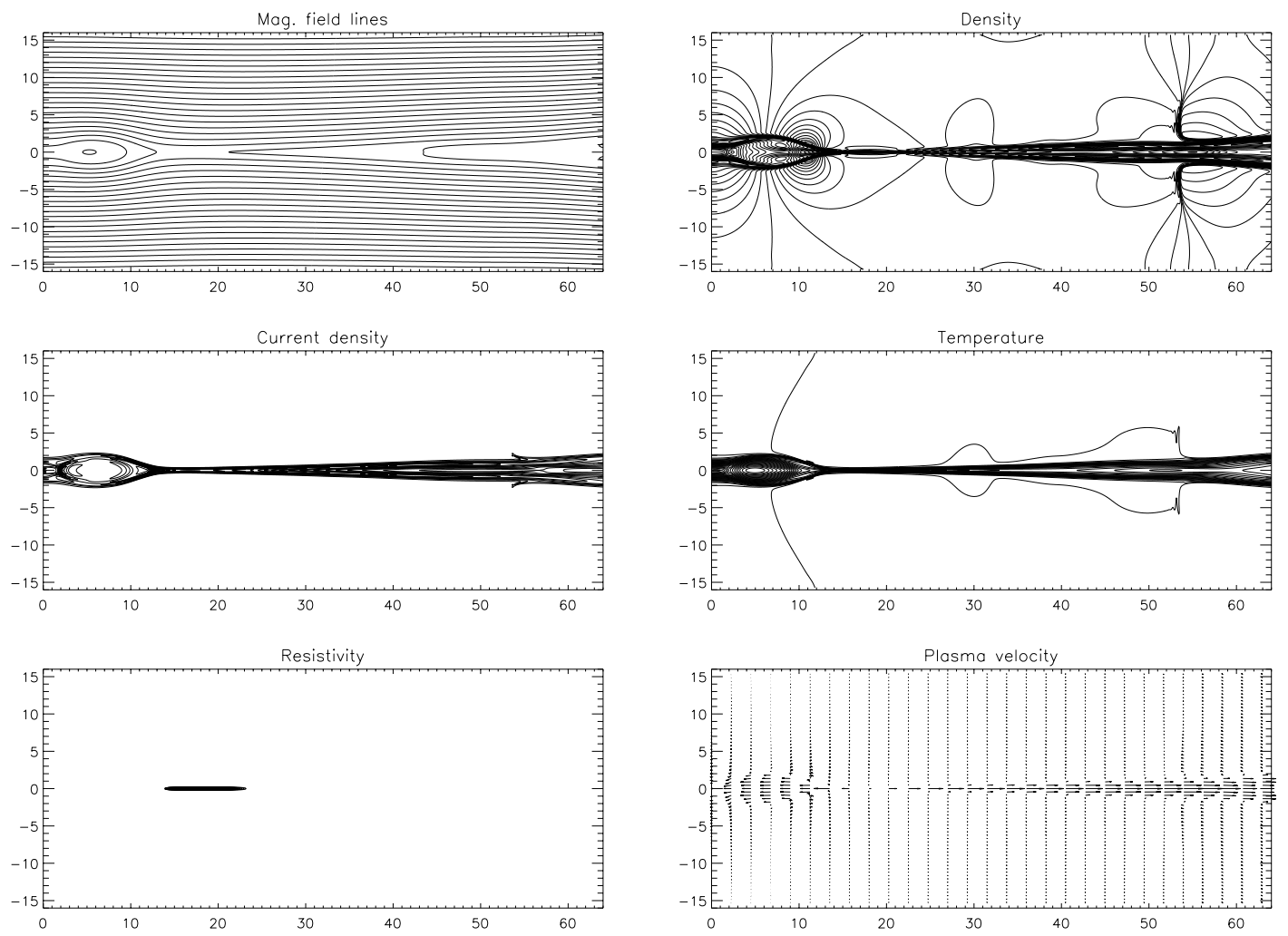

Fig. 6. Magnetic field lines, current density, places of enhanced electric resistivity, plasma density and temperature isolines and velocity field during the model reconnection process at $280 \tau_{\mathrm{A}}$.

the number of degrees of freedom (in our 2-D case the number $N=2$ ). The electric field is given by

$\boldsymbol{E}=-\boldsymbol{u} \times \boldsymbol{B}+\eta \boldsymbol{j}$.

A current sheet with antiparallel magnetic field and uniform density is chosen for the initial state:

$\boldsymbol{B}=-B_{0} \tanh \left(y / l_{\mathrm{CS}}\right) \boldsymbol{e}_{x}$,

$\boldsymbol{u}=0$,

$\rho=\rho_{0}$,

$p=(1+\beta) B_{0}^{2} /\left(2 \mu_{0}\right)-B_{x}^{2} /\left(2 \mu_{0}\right)$,

where the plasma beta is $\beta=2 \mu_{0} p(|y| \rightarrow \infty) / B_{0}^{2}$.

All the following computations are made in normalized variables derived from the current sheet half width $l_{\mathrm{CS}}$ and the asymptotic $(|y| \rightarrow \infty)$ Alfvén velocity $V_{A}=B_{0} /\left(\mu_{0} \rho_{0}\right)^{1 / 2}$ of the configuration at $t=0$. Time is measured in units of the Alfvén time $\tau_{\mathrm{A}}=l_{\mathrm{CS}} / V_{A}$, and $p, \boldsymbol{E}, \boldsymbol{j}$, and $\eta$ are normalized by $B_{0}^{2} /\left(2 \mu_{0}\right), V_{A} B_{0}, B_{0} /\left(\mu_{0} l_{\mathrm{CS}}\right)$, and $\mu_{0} l_{\mathrm{CS}} V_{A}$, respectively.

At the initial state the localized resistivity

$\eta\left(x, y, t \leq t_{0}\right)=0.02 \exp \left[-(x / 0.8)^{2}-(y / 0.8)^{2}\right]$,

(fixed for a few Alfvén times, $t_{0}=10$ ) is included to start the reconnection processes. Thereafter the resistivity is variable and is determined self-consistently at each grid point and time step from the electron-ion drift velocity $v_{\mathrm{D}}=v_{0} j / \rho$ (where the factor $v_{0}=v_{\mathrm{D}}(0)=d_{\mathrm{i}} / l_{\mathrm{CS}}$ results from the normalization and $d_{\mathrm{i}}=c / \omega_{\mathrm{pi}}$ is the ion inertial length). An "anomalous" value, $\eta_{\mathrm{an}}$, is set if the threshold, $v_{\mathrm{cr}}$, is exceeded, otherwise vanishing resistivity is assumed:

$\eta(\boldsymbol{x}, t)=\left\{\begin{array}{lll}0 & : & \left|v_{\mathrm{D}}\right| \leq v_{\mathrm{cr}} \\ C \frac{\left(\left|v_{\mathrm{D}}(\boldsymbol{x}, t)\right|-v_{\mathrm{cr}}\right)}{v_{0}} & : & \left|v_{\mathrm{D}}\right|>v_{\mathrm{cr}} .\end{array}\right.$

Here $C=0.003$ and $v_{\text {cr }}=3 v_{0}$. A two-step Lax-Wendroff scheme and a uniform Cartesian grid with resolutions $\Delta x=$ $\Delta y=0.045$ are employed for numerical integration. The plasma beta is 0.15 . For the stabilization the artificial smoothing in the form:

$\boldsymbol{\Psi}_{i j}^{n} \longrightarrow \lambda \boldsymbol{\Psi}_{i, j}^{n}+\frac{1-\lambda}{4}\left(\boldsymbol{\Psi}_{i+1, j}^{n}+\boldsymbol{\Psi}_{i-1, j}^{n}+\boldsymbol{\Psi}_{i, j+1}^{n}+\boldsymbol{\Psi}_{i, j-1}^{n}\right)$,

where $\lambda$ is the variable smoothing parameter and $\boldsymbol{\Psi}$ represents $\left(\rho, \rho u_{x}, \rho u_{y}, B_{x}, B_{y}, U\right)$ variables, is used. Computations are performed in the numerical box $\langle 0 ; 64\rangle \times\langle 0 ; 16\rangle$ with symmetrical boundary conditions used at the lower and left boundaries; external box boundaries are free.

Computations were made for 1200 Alfvén transit times $\tau_{\mathrm{A}}$ and results are shown in Figs. 6-8. Figures 6 and 7 present states of the magnetic field reconnection (magnetic field lines, current density, electric resistivity, plasma density and temperature isolines, and plasma velocity field) at $t=280$ and $t=980 \tau_{\mathrm{A}}$, respectively. In Fig. 6 one magnetic island (plasmoid) and reconnection plasma outflow can be seen in the current sheet at positions $x \sim 5, x=20-64$, respectively. The energy release is concentrated at regions of high resistivity (position $x=13-23$ ). For longer times, a large plasmoid is formed at around $x=0, y=0$, and all dissipative processes are shifted 

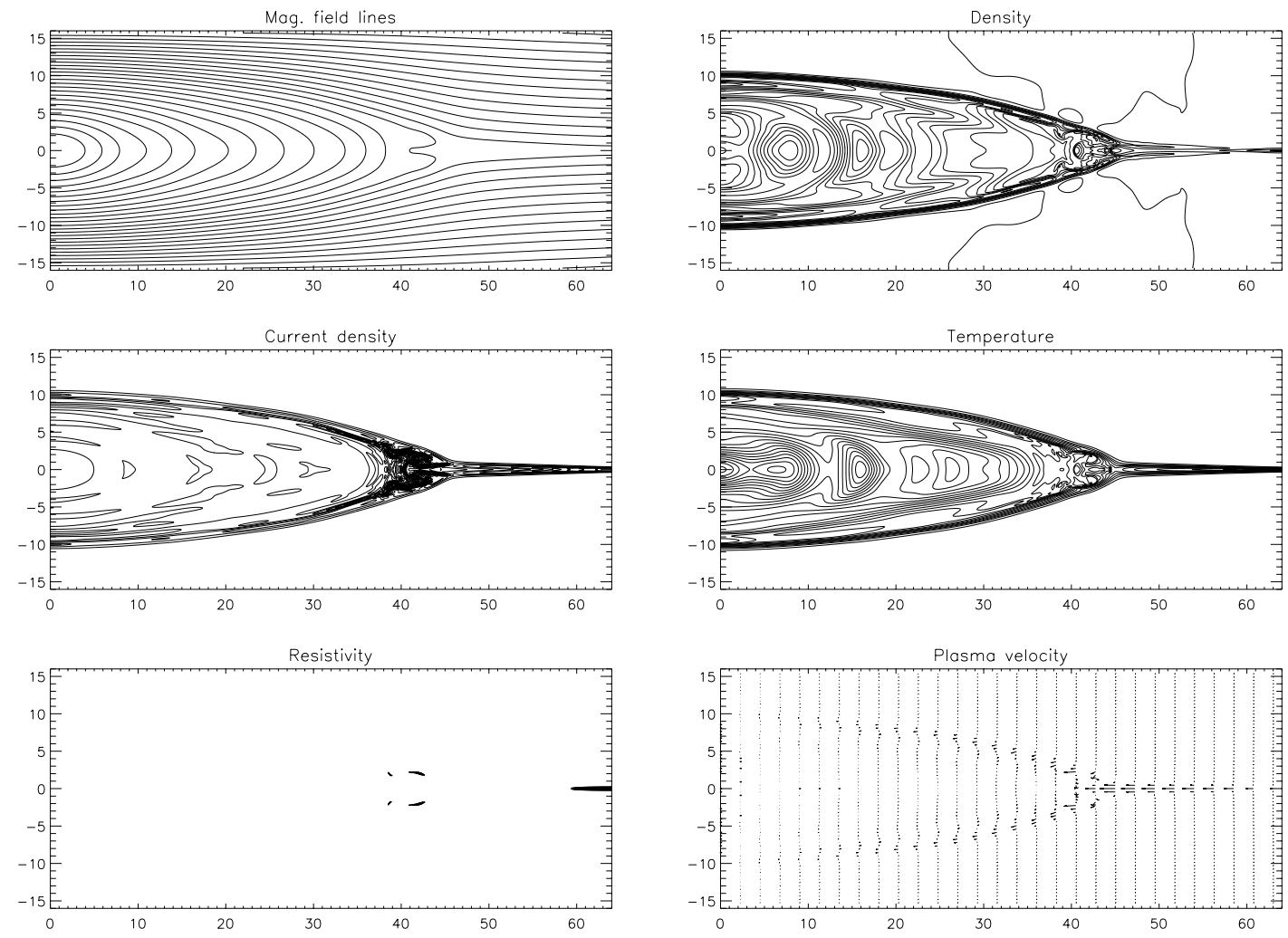

Fig. 7. Magnetic field lines, current density, places of enhanced electric resistivity, plasma density and temperature isolines and velocity field during the model reconnection process at $980 \tau_{\mathrm{A}}$.
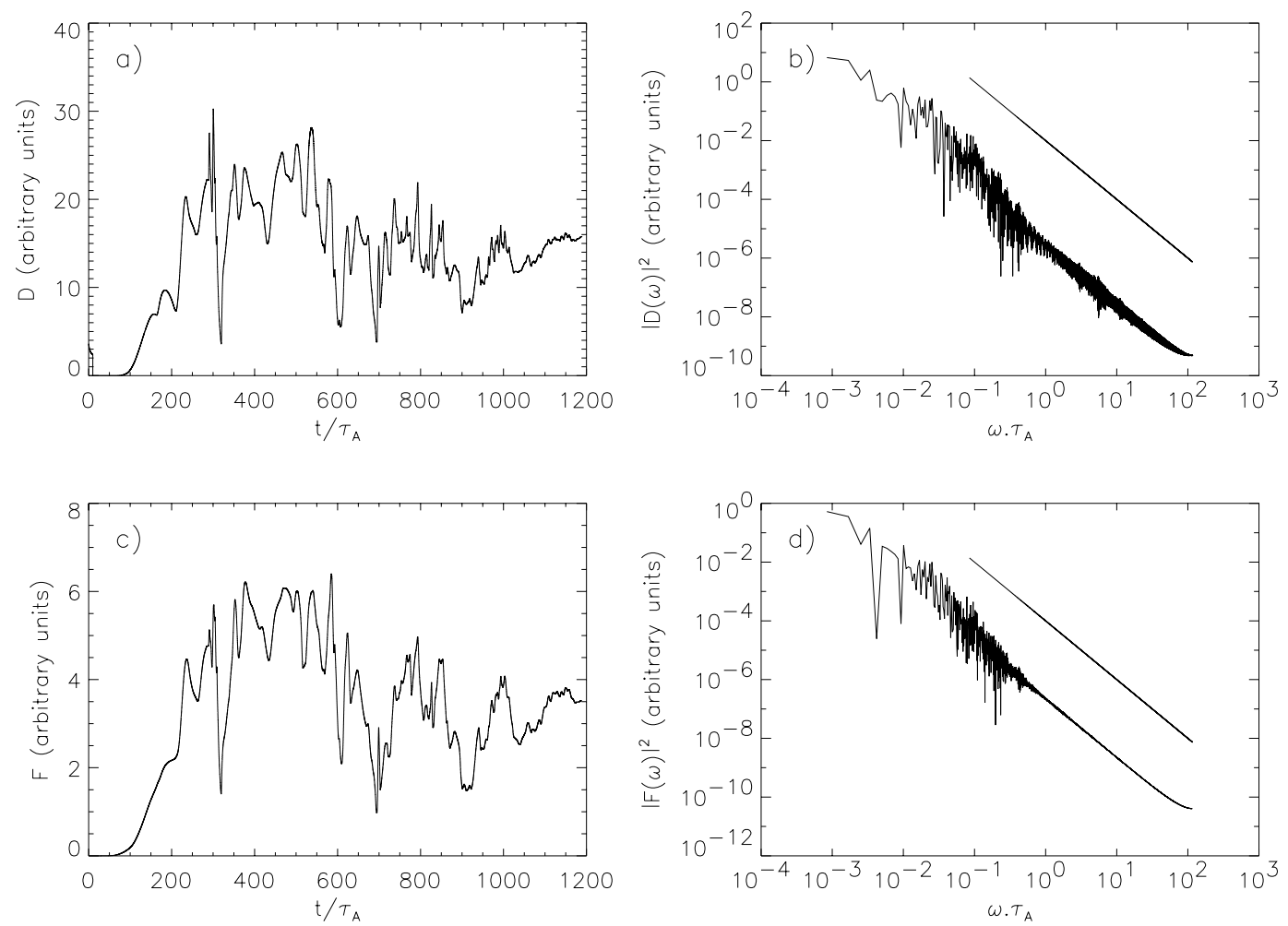

Fig. 8. Time variations of the dissipation power $D$ computed by the 2-D MHD numerical model a), their Fourier power spectrum b), time variations of the radio radiation measure $(\mathrm{F}) \mathbf{c})$, their Fourier power spectrum d). $\tau_{\mathrm{A}}$ is the Alfvén transit time. In plots $\left.\mathbf{b}\right)$ and d) the line corresponding to the power-law spectrum with power-law index 2 is added for comparison. 
to greater values of $x$ (Fig. 7) (and symmetrically on the opposite side of the plasmoid at negative values of $x$ ).

\subsection{Comparison with radio flux variations}

In order to compare directly the dynamical characteristics of the modelled reconnection process with those obtained from the observed radio data the temporal evolution of two integral quantities was traced. The first one is the integral dissipated power

$D=\int_{\text {Box }} \eta(x, y) j^{2}(x, y) \mathrm{d} x \mathrm{~d} y$.

The second one - radio radiation measure - roughly estimates the radio flux emitted from the reconnection box and is computed in the following way: as mentioned earlier, the radio pulsations are supposed to be due to electron beams accelerated in the reconnection DC electric field $E=\eta j$. The ratio of beam to background densities $n_{\mathrm{b}} / n_{0}$ - the relative number of electrons decoupled from the collisional thermal bulk (runaway electrons) - is approximately given by (Heyvaerts 1981):

$\frac{n_{\mathrm{b}}}{n_{0}} \approx \frac{1}{2} \exp \left(-\frac{1}{2} \frac{E_{\mathrm{D}}}{E}\right)$

where

$E_{\mathrm{D}}=\frac{m v_{T_{\mathrm{e}}}}{e \tau_{\mathrm{c}}}=n e v_{T_{\mathrm{e}}} \eta$

is the Dreicer electric field, $\tau_{\mathrm{c}}$ being the electron collision time. This concept is not limited to inter-particle collisions only, but remains valid also for other mechanisms of resistivity, provided similar velocity dependence on the collisional frequency (Heyvaerts 1981). It is reasonable to suppose rather narrow (in velocity space) electron beams in accordance with this acceleration mechanism. Thus, the reactive version of the bump-in-tail instability should apply in the plasma emission process with the growth rate (Melrose 1980)

$\gamma=\alpha\left(\frac{n_{\mathrm{b}}}{n_{0}}\right)^{1 / 3} \omega_{\mathrm{pe}}$

Here, $\alpha$ is a factor of order of unity and $\omega_{\mathrm{pe}}$ is the electron plasma frequency. The sum of this growth rate over all grids of the computation box was then chosen as a rough measure of the radio emission. Combining Eqs. (19) and (20) and after some renormalisation it follows that

$F=\int_{\text {Box }} \exp \left(-\frac{1}{6} \sqrt{\frac{M}{m}} \frac{v_{\text {cr }}}{v_{0}} \frac{\rho(x, y)}{j(x, y)}\right) \mathrm{d} x \mathrm{~d} y$,

where $v_{\text {cr }}$ and $v_{0}$ are defined by relation (18).

This analysis has shown that the reconnection process is strongly variable (intermittent, bursting) in time (see Fig. 8) where the time variations of the spatially integrated dissipation power $D$ and the radio radiation measure $F$ are presented.

Finally, we prepared the Fourier power spectra of these variations (see b) and d) plots in Fig. 8). Both have a powerlaw form with a power-law index of 2.

\section{Discussion and conclusions}

In the present paper, the model of drifting pulsating structures proposed by Kliem et al. (2000) was generalized by a concept of multi-scale (cascading) reconnection. This concept agrees with power-law dependencies found in observed radio fluxes and numerical simulations.

Analyzing the high-time resolution $(1 \mathrm{~ms})$ observations of the slowly drifting pulsating structure at 1420 and $610 \mathrm{MHz}$ we found periods in the range of seconds (0.9-7.5 s, see Table 1). In shorter periods, Fourier power spectra show a power-law form, especially in the $0.06-0.2 \mathrm{~s}$ range, where the power-law indices were in the interval 1.3-1.6.

The slowly drifting pulsating structure was interpreted as plasma radiation at fundamental plasma frequency, which is supported by its partial polarization. We proposed, in agreement with our previous papers, that pulses in the pulsating structures manifest electron beams accelerated in the multi-scale magnetic field reconnection and their trapping in magnetic field islands (plasmoids). We propose that when the plasmoids move upwards in the solar atmosphere, the plasma density outside and inside decreases and thus the radio emission of pulses as a whole drifts slowly towards lower frequencies.

We combined the ideas of Shibata \& Tanuma (2001) and Tajima et al. (1987) and estimated the time scales in the multiscale reconnection as

$t^{n} \approx 2 \pi \beta^{3 / 2} \frac{L_{n}}{v_{\mathrm{A}}}$,

where $L_{n}$ represents spatial scales given by the relation (5). Thus, for example the found period of $7.5 \mathrm{~s}$ gives the characteristic length $L=38000 \mathrm{~km}$, for $\beta=0.1$ and Alfvén speed $v_{\mathrm{A}}=1000 \mathrm{~km} \mathrm{~s}^{-1}$. Clearly, shorter periods correspond to smaller spatial scales in the multi-scale reconnection.

Using the 2-D MHD model we simulated the proposed reconnection processes and we found that Fourier power spectra of variations of the dissipation power and of the radio radiation measure have a power-law form with a power-law index of 2. This result is an extension of the results of Valdivia et al. (2003), who made similar computations, but in the 1-D model only. They found a Fourier spectrum with a power-law index of 1 . Thus, we expect that in reality, i.e. in the 3-D case, the Fourier power spectrum also has a power-law form, but with a power-law index $\geq 2$. These results show that tearing and coalescence processes in multi-scale reconnection are self-similar, i.e. they do not have a characteristic scale length.

Ryabov et al. (1997) studied fine structures in 2.5 and $2.85 \mathrm{GHz}$ radio bursts and they found power-law Fourier spectra with a power-law index in the range $1-2$, which is similar to our case (1.3-1.6). They suggested a model of uncorrelated pulses as capable of generating such spectra. Thus, the proposed multi-scale reconnection processes may belong to the same class of processes. On the other hand, deviations from power-law dependence can be expected for longer time scales (corresponding to larger spatial scales) due to the limited volume of the flare. For longer period ranges we found well-specified periods of $0.9-7.5 \mathrm{~s}$ (Table 1 ). 
Thus, the difference in the power-law indices of Fourier spectra of the observed and of the simulated data are likely due to non-linear processes, which in reality connect the dissipation power in the reconnection and the radio flux output, i.e. dissipation power $\rightarrow$ electron acceleration $\rightarrow$ plasma wave generation $\rightarrow$ transformation of the plasma waves into the electromagnetic ones $\rightarrow$ absorption of the electromagnetic waves.

The results of the statistical analysis of the observed data, which indicate high-dimensions, non-linear and stochastic processes, agree with the proposed multi-scale reconnection process.

Acknowledgements. This research was supported by Grant IAA3003202 of the Academy of Sciences of the Czech Republic. The authors thank Andreas Klassen for providing the Potsdam radio spectrum.

\section{References}

Heyvaerts, J. 1981, in Solar Magnetohydrodynamics, ed. E. R. Priest (New York: Gordon and Breach Science Publishers), 429

Hudson, H. S., Kosugi, T., Nitta, N., \& Shimojo, M. 2001, ApJ, 561, L211
Karlický, M. 1988, Bull. Astron. Czech., 39, 13

Karlický, M. 2004, A\&A, 417, 325

Karlický, M., \& Odstrčil, D. 1994, Sol. Phys., 155, 171

Karlický, M., Fárník, F., \& Mészárosová, H. 2002, A\&A, 395, 677

Khan, J. I., Vilmer, N., Saint-Hilaire, P., \& Benz, A. O. 2002, A\&A, 388,363

Klassen, A., Pohjolainen, S., \& Klein, K.-L. 2003, Sol. Phys., 218, 197

Kliem, B., Karlický, M., \& Benz, A. O. 2000, A\&A, 360, 715

Kundu, M. R., Nindos, A., Vilmer, N., et al. 2001, ApJ, 559, 443

Melrose, D. B. 1980, Plasma Astrophysics (New York: Gordon and Breach Science Publishers)

Mészárosová, H., Veronig, A., Zlobec, P., \& Karlický, M. 2003, A\&A, 407, 1115

Priest, E. R., \& Forbes, T. 2000, Magnetic Reconnection (Cambridge, UK: Cambridge University Press), 177

Ryabov, V. B., Stepanov, A. V., Usik, P. V., et al. 1997, A\&A, 324, 750

Schumacher, J., \& Kliem, B. 1996, Phys. Plasmas, 3, 4703

Shibata, K., \& Tanuma, S. 2001, Earth Planets Space, 53, 473

Tajima, T., Sakai, J. I., Nakajima, H., et al. 1987, ApJ, 321, 1031

Tanuma, S., Yokoyama, T., Kudoh, T., \& Shibata, K. 2001, ApJ, 551, 312

Valdivia, J. A., Klimas, A., Vassiliadis, D., Uritsky, V., \& Takalo, J. 2003, Space Sci. Rev., 107, 515 